\title{
Informatización del inventario de cementerios andaluces
}

\section{Carmen Guerra de Hoyos}

Arquitecta

La sistematización e informatización del inventario de cementerios es un trabajo que surge de la colaboración entre el Instituto del Patrimonio Histórico y la Consejería de Obras Públicas de la Junta de Andalucía, debido al interés existente en adecuar una gran cantidad de información perteneciente a diversas investigaciones y trabajos de campo realizados por la Consejería de Obras Públicas a un formato de trabajo que mejore su accesibilidad y facilite su estudio. Se trata pues de la primera experiencia en esta labor conjunta para la que se elige el Inventario de Cementerios Andaluces, por tratarse de una investigación bastante acotada, en la que se podrían aquilatar tanto los requerimientos necesarios para este tipo de trabajo como los resultados obtenidos.

El inventario de cementerios surge como encargo efectuado por la Consejería de Obras Públicas a Eduardo Ortiz, arquitecto, en 1987, para realizar una catalogación sobre un tipo de inmueble del que no se conocían muchos datos. Se planteó como una investigación bastante dilatada en el tiempo, aproximadamente tres años, durante los cuales se realizó el trabajo de campo, que dio como fruto un conjunto de 178 fichas representativas de las ocho provincias andaluzas. Se contemplaban como cementerios a catalogar los que presentasen características de interés por su emplazamiento, relación con el entorno natural o urbano, su ordenación o los tipos de enterramiento que albergasen.

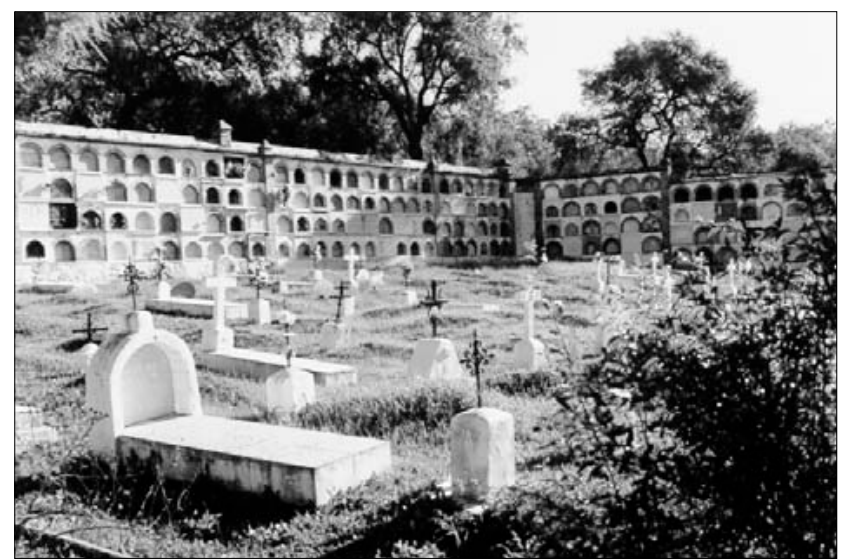

Posteriormente, sobre esta investigación se organiza por la misma Consejería una exposición y una publicación llamadas "Cementerios Andaluces", que entre otros aspectos recogían una planimetría y fotogafías detalladas de 28 de los cementerios catalogados en el inventario.

De estas dos fuentes documentales parte el trabajo de sistematización e informatización, que ha consistido en el trasvase de la información recogida por el inventario a la base de datos SIBIA. Se ha contemplado como un proceso que hiciera accesible un conjunto de datos que, de otra manera, resultaría bastante difícil de manejar, en este caso concreto, principalmente por la heterogeneidad de los mismos, puesto que el prolongado desarrollo de la investigación en el tiempo ha supuesto la evolución de los criterios en la recogida de datos.

Por otro lado la base de datos utilizada es un instrumento de almacenaje de información superior al requerido por las fichas base del inventario. Esto ha determinado un acoplamiento entre ambos elementos, que ha llevado a simplificar la base de datos original en los campos necesarios para la descripción de la información recogida y a reorganizar la información de las fichas para adaptarse a la sistematización de SIBIA.

Hay que reseñar que durante este proceso se ha procurado garantizar que la práctica totalidad de los datos contenidos en el inventario se ha volcado en la base de datos, a excepción de aquellos que, por tratarse de referencias temporales, datos de los que no se puede constatar su vigencia o por no ser factible su informatización, no han podido ser incluidos.

El resultado ha sido un conjunto de 178 fichas de cementerios, a 28 de las cuales, además de la información contenida en el inventario se las ha complementado con planimetría y fotografías realizadas para la 


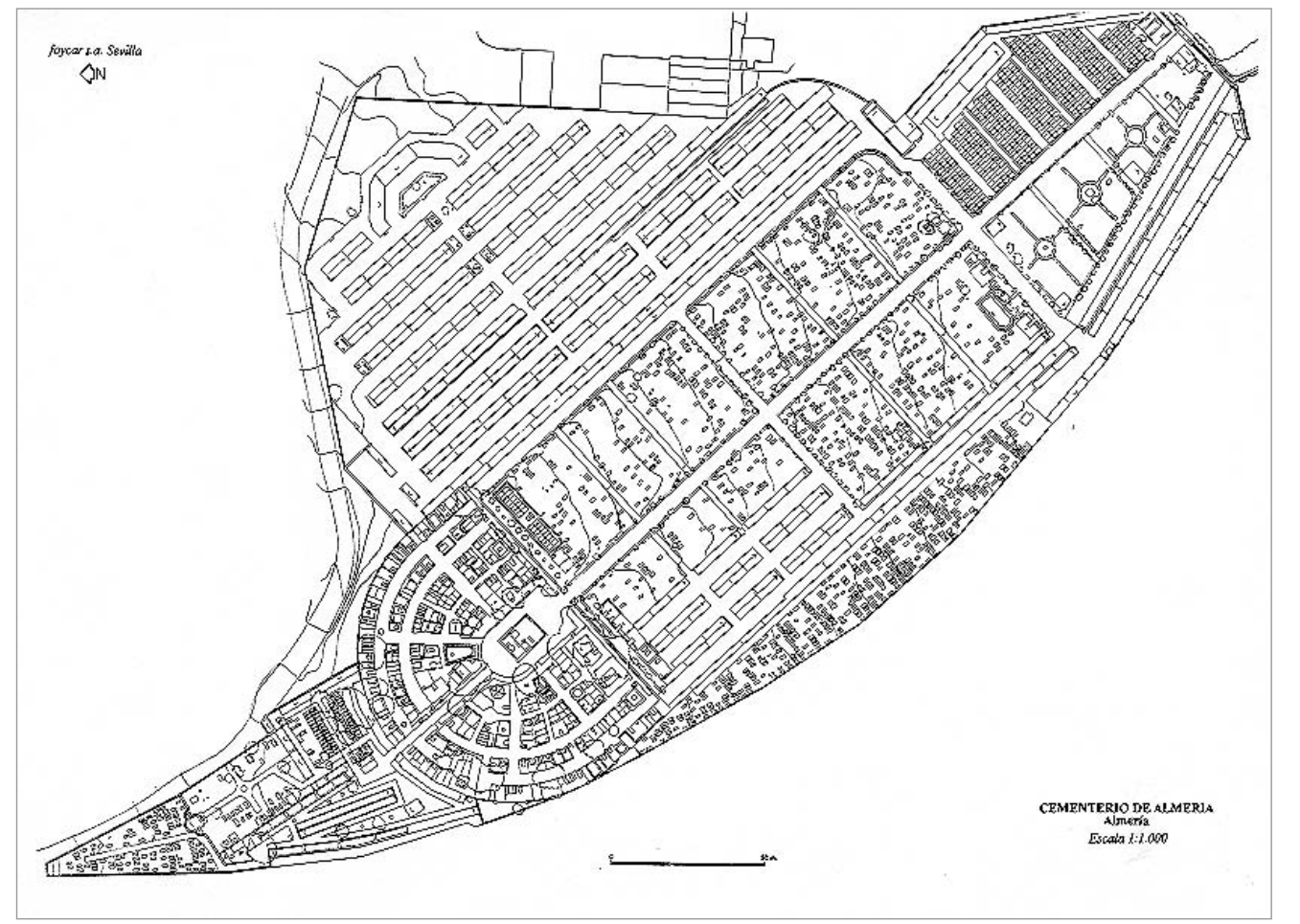

publicación y exposición "Cementerios de Andalucía. Para terminar se ha efectuado la georeferenciación de los elementos inventariados en el programa Arcview, al objeto de incorporarlos a las coberturas de información patrimoniales del Centro de Documentación. Estas coberturas posibilitan el análisis espacial del Patrimonio arquitectónico andaluz.

Quizás la ventaja más apreciable que supone la informatización del inventario y su incorporación a programas de localización, es que de unos elementos de los que, aunque constatado su interés, no era factible su disponibilidad, ahora es posible su fácil acceso desde tantos medios como a los que dan servicio las bases de datos del Instituto del Patrimonio Histórico.

Pero también se puede contar con los resultados "secundarios" del trabajo, pues al reorganizar los datos del conjunto de fichas se ha efectuado una labor de revisión y análisis sobre los elementos inventariados. Esto ha permitido aventurar un conjunto de divisiones tipológicas sobre tipos de cementerios y tipos de enterramientos catalogados. Hay que tener en cuenta que estos análisis se realizan sobre el material del que disponemos, y aunque las conclusiones sean sobre los datos del inventario, revelan pautas bastantes abarcativas del conjunto de cementerios andaluces.

\section{Tipologías de cementerios}

Se establece una división primaria entre cementerios que disponen de un recinto único, con un espacio central, dividido o no en cuarteles por ejes o paseos, rodeado por una cerca en la que se suelen disponer enterramientos en nichos, y un segundo grupo de cementerios que además de este espacio posiblemente inicial, han sufrido una o más ampliaciones, normal-

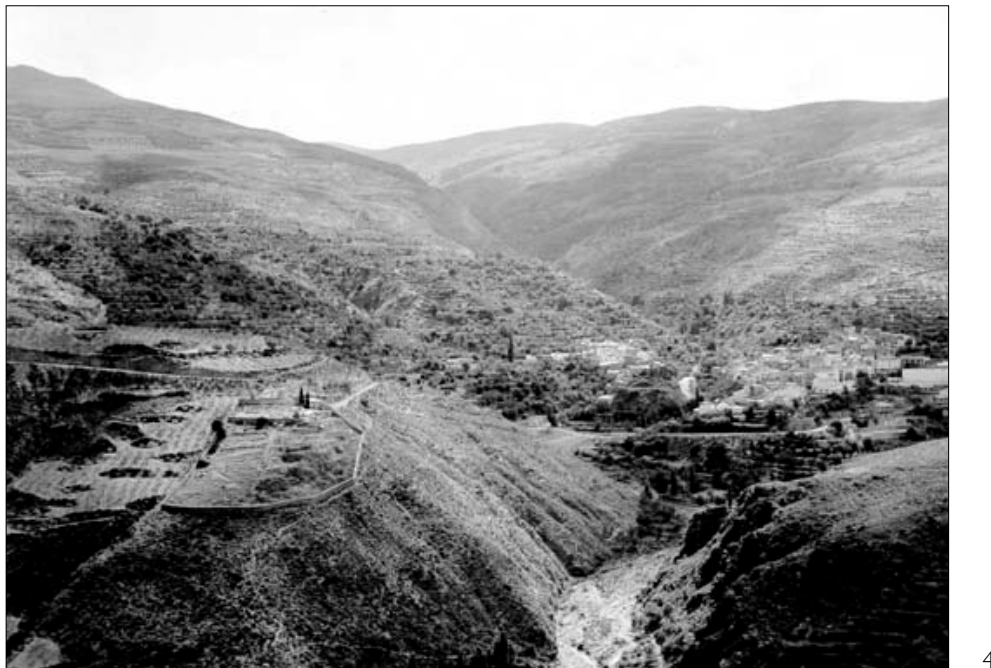

mente laterales, que han desvirtuado la imagen de conjunto homogéneo. Tenemos así las dos primeras categorías, cementerios de I recinto y cementerios de varios recintos. Esta división nos permite separar en principio cementerios más alterados de aquellos que han sufrido pocos cambios, bien porque dispongan de espacio suficiente para los enterramientos o por que hayan sido suplementados por otros cementerios cercanos.

Además se cuenta con otros tipos de cementerios que responden a implantaciones muy concretas: el topográfico, el monumental, el cementerio jardín y el cementerio con cuevas.

Se ha considerado cementerio topográfico a aquel cuya característica fundamental es adaptarse a una ubicación escalonada, siendo esta circunstancia la que condiciona la ocupación del cementerio. En este 

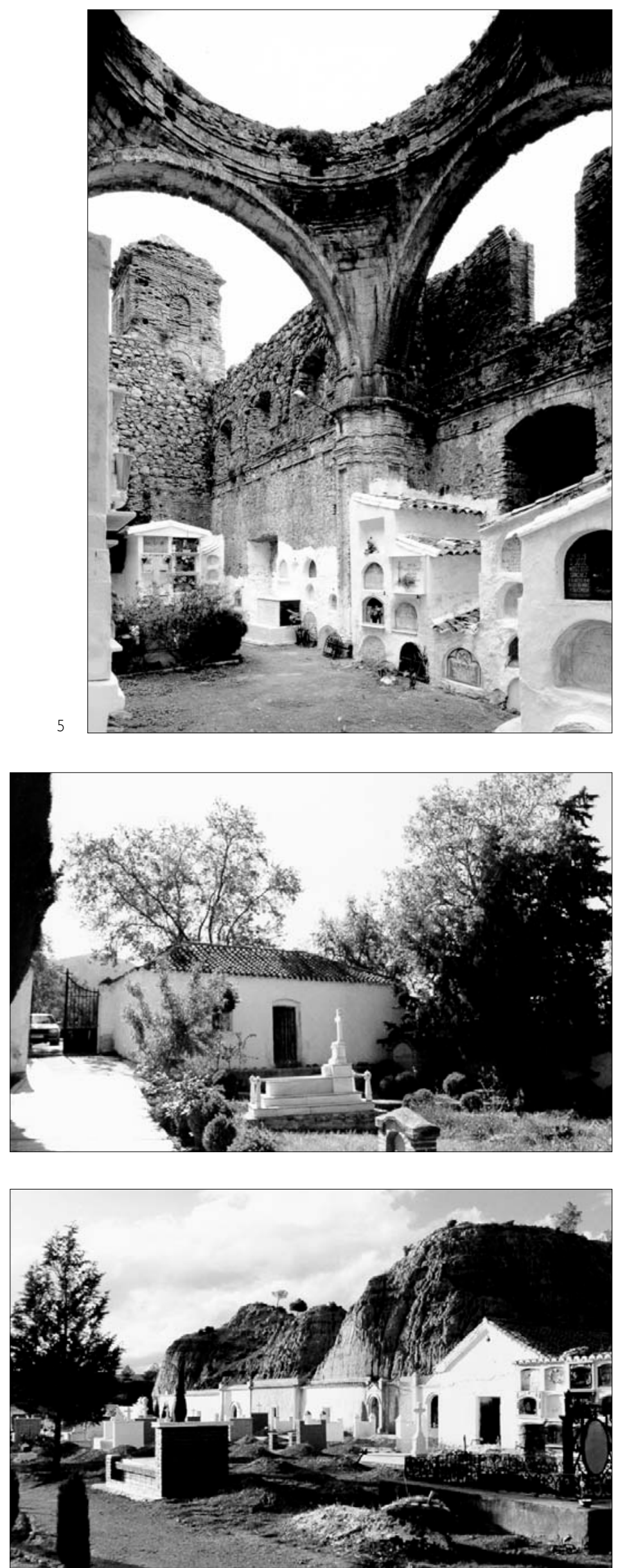

grupo se pueden encontrar cementerios "de montaña", que apenas se distinguen del entorno (Bacares), o cementerios que se pegan a la falda de un monte y se disponen según las curvas de nivel (Zahara, Vilches), a otros cementerios que reproducen una estructura urbana de calles en cuesta y ocupación muy densa (Martos).

Como cementerio monumental consideramos al que se encuentra dentro de un recinto edificado previo, de interés, independientemente del cementerio, el caso más frecuente es el de cementerio alojado en recinto amurallado, pero también hay un cementerio alojado en una iglesia ruinosa (Villaluenga del Rosario). Los cementerios adosados a edificios, normalmente iglesia o ermitas, caso bastante frecuente, no se han incluido en este grupo al no estar tan condicionados por el monumento como los anteriores, sino que se han recogido por el número de recintos. Normalmente disponen de un solo recinto.

Se ha considerado cementerio jardín aquel en el que la vegetación pasa de ser un motivo de interés a convertirse en un factor vertebrador de la ordenación. Con ejemplos que van desde un cementerio con influencia de la cultura anglosajona como el cementerio Británico de Málaga, a cementerios en los que se cultivan árboles frutales. Aunque es un tipo muy poco frecuente no deja de resultar interesante su contraste con el resto de cementerios inventariados.

Como última variante se ha recogido el cementerio con cuevas, de los cuales se inventarían cuatro en la provincia de Granada. En primer lugar porque su ubicación junto a colinas o laderas que se excavan para efectuar los enterramientos da una imagen muy concreta y característica. En segundo porque los tipos de enterramientos que alberga (cuevas, en suelo o mixtos) adquieren técnicas y repertorios formales propios que merecen que, además de separarse como tipo de enterramiento característico, se les considere como una implantación tipológicamente independiente.

\section{Enterramientos}

En cuanto a los tipos de enterramiento hay que señalar que se ha establecido una simplificación bastante drástica porque la descripción recogida en las fichas es tan variada que hace casi imposible la clasificación de los datos.

Por ello se han separado los tipos de enterramiento de interés en los siguientes grupos: mausoleos y panteones, bóvedas trasdosadas, grupos de nichos, sepulturas en suelo, enterramientos en cueva, y enterramientos bajo rasante.

En el grupo de mausoleos y panteones se incluyen todos los enterramientos independientes, de considerables dimensiones, adosados o no, con habitáculo, con edículo, etc, que el autor del inventario ha considerado dignos de interés. En algunas ocasiones 
esta consideración se debe al repertorio formal y la calidad artística del elemento, en otras a los materiales utilizados y en otras a la disposición u ordenación de los elementos entre sí (panteones adosados).

Las bóvedas trasdosadas son un tipo de enterramiento muy concreto, en el que la parte superior se curva en bóveda, reflejando una cierta imagen antropomórfica. Se trata de un tipo de enterramiento que tiende a desaparecer, junto a sus técnicas constructivas, por lo que el autor manifiesta un palpable interés por reseñarlo. Hay casos de bóvedas trasdosadas que aparecen en hileras, en agrupaciones de varias alturas o en situación espontánea.

El conjunto de "grupos de nichos" engloba un grupo bastante amplio de actuaciones, que abarca desde la agrupación de varios enterramientos en altura, formando un edículo con tejado y/o vestíbulo, a nichos que se disponen bajo un pórtico o soportal, incluso se incluyen cuando es de interés un elemento característico de las agrupaciones, como las cresterías, los remates etc, también cuando la disposición de los nichos es interesante.

Las sepulturas en el suelo suelen reseñarse bien por tener un elemento emergente que las caracterice, como una cartela, un dado o una hornacina, o bien por el recubrimiento efectuado del féretro, con piedras, en forma antropomórfica, o en mastaba.

Los enterramientos en cueva se efectúan de manera muy parecida a como se realizan las excavaciones para las viviendas en cuevas. Constituyen un pequeño grupo de casos pero de mucho interés, tanto por los espacios y la imagen que generan, como por ser representativos de un modo de habitar muy especifico de la zona donde se localizan.

Enterramientos bajo rasante se han denominado a unos enterramientos especiales y muy concretos que no responden a las características comunes al resto de enterramientos. Se trata de unos enterramientos en grupo excavados bajo rasante, originados por una normativa local o por el descubrimiento de unas galerías romanas (Monturque). Paradójicamente, en el primer caso los enterramientos sobre rasante se reservaban para clases acomodadas y en el segundo los enterramientos en las galerías fueron destinados en principio para gente "pudiente".

\section{Conclusiones.}

Hay que reseñar que la información recogida en las fichas supone una amplia panorámica sobre un tipo de inmueble del que no existe mucha información concreta, y que aporta una perspectiva muy válida sobre las culturas que los originan, convirtiéndose fundamentalmente en la imagen que quieren perpetuar de ellas mismas.

Hay que destacar la riqueza y variedad del material elaborado, que abarca desde intervenciones de arqui-
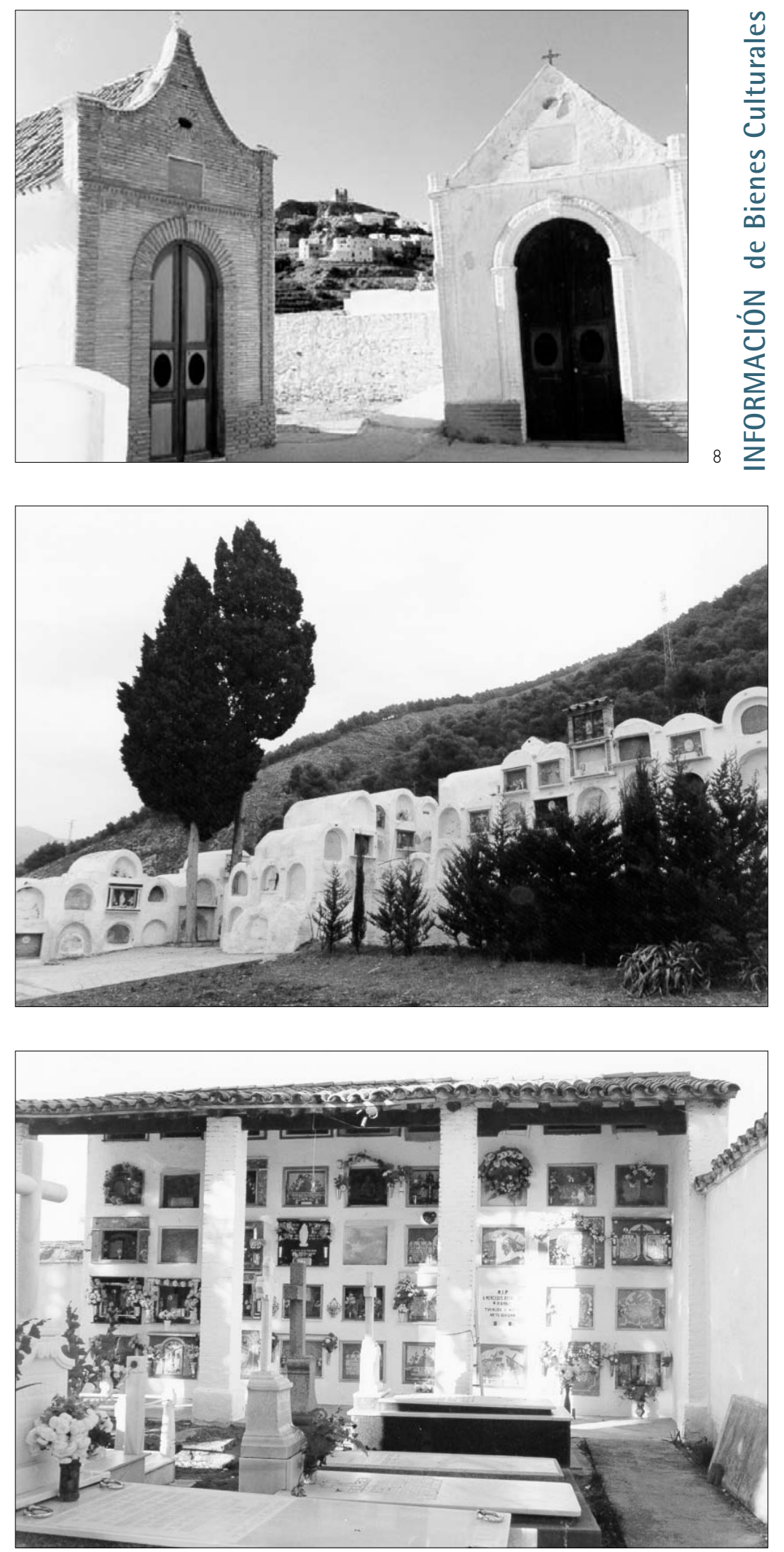

5. Cementerio monumental. Villaluenga del Rosario. Cádiz.

6. Cementerio jardín. Nigüelas. Granada.

7. Cementerio con cuevas. Benalúa de Guadix. Granada

8. Mausoleos y Panteones. Canjayar. Almería.

9. Bóvedas trasdosadas. Alcaucín. Málaga.

10. Grupos de nichos. Adamuz. Córdoba. 
tectura culta, no necesariamente ligadas a poblaciones grandes, a cementerios enormemente primitivos que disponen los enterramientos de una manera espontánea, o incluso enterramientos trogloditas, allí donde se utilizan cuevas como viviendas. Cementerios que forman parte del entorno urbano, otros que se encuentran en situación aislada con implantación destacada en el territorio, o que se funden con la orografía, incluso incluyendo arbolado y vegetación del entorno. Nos encontramos con la utilización de elementos o edificios de valor histórico, artístico o arqueológico, con cementerios ligados a los modos de producción de la zona donde se asientan, como los de zonas mineras. Implantaciones unitarias en el tiempo o cementerios de larga consolidación, reformados, rehabilitados y ampliados en épocas sucesivas.

Quizás lo más interesante es la cantidad de vías de investigación que pueden abrirse desde este primer acercamiento. En primer lugar y por que están ligados
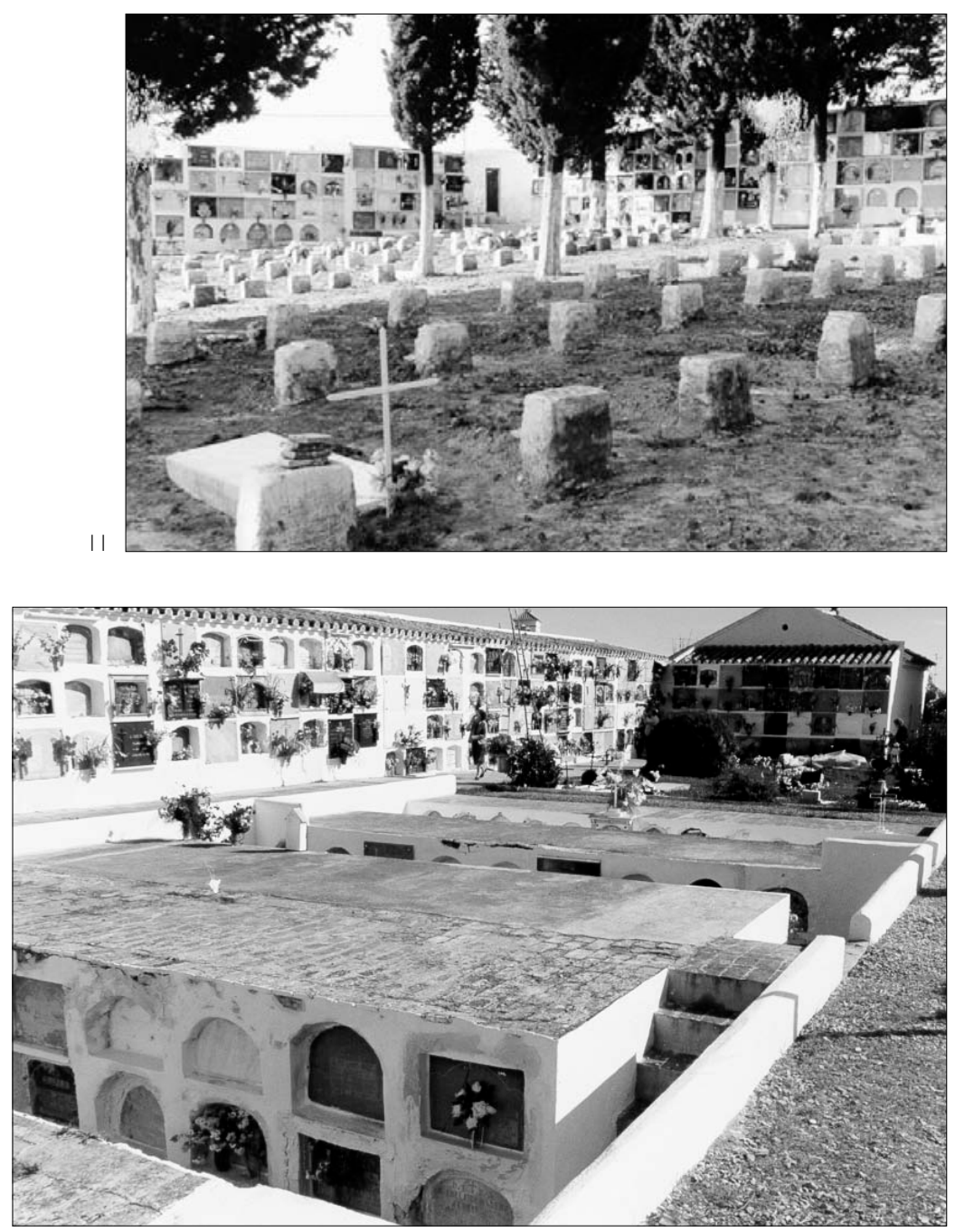

a técnicas constructivas locales, en proceso de desaparición, resultan muy interesante los tipos tradicionales de enterramientos, de bóveda trasdosada, agrupados o no, en edículos de varias alturas con corral y bancos previos, etc. En la actualidad la inhumación en nichos estandarizados está erradicando esta riqueza formal de los cementerios. También sería de interés una profundización en los diferentes espacios generados por la ocupación funeraria, y su resultado, con el fin de introducir más variables tipológicas en la categoría de cementerios, que reflejase la enorme diversidad existente. La relación entre repertorios formales escogidos y el desarrollo histórico de la población, el reflejo que se produce de la forma de la ciudad en la morfología del cementerio, en ocasiones muy claro, sobre todo cuando algún factor como la topografía lo potencia, o la elaboración de códigos formales propios adaptando la simbología tradicional a gustos locales. $\bigcirc$ entre técnicas constructivas y medios de producción de la zona, por ejemplo la utilización de cantería en panteones de las provincias de Jaén y Almería.

Por último, y como campo independiente, reseñar la enorme riqueza que supone todo el repertorio de leyendas, mitos, costumbres y ritos asociados a los cementerios. El lapidario y su epigrafía, la relación entre el tipo de enterramiento y la situación social del personaje, etc. Hay que reseñar que en algunas de las fichas se reseñaban los nombres de los enterramientos de interés, los de los enterramientos más antiguos y enterramientos de personajes destacados de la población. Estos datos han sido recogidos en su totalidad por su posible aportación de información sobre la estructura social local de las poblaciones.

Sin duda el acercamiento que supone la informatización de estos datos a los investigadores facilitará la realización de numerosos e interesantes trabajos.

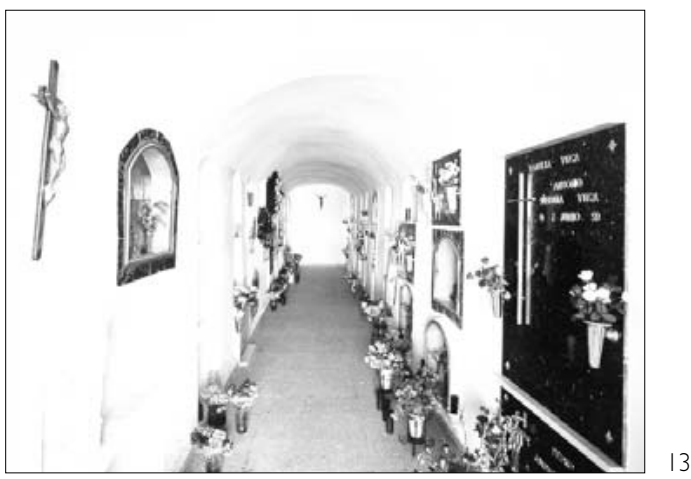

II. Sepulturas en el suelo. Villanueva del Río y Minas. Sevilla.

12. Enterramientos en cueva. Benalúa de Guadix. Granada.

13. Enterramientos bajo rasante. Hornachuelos. Córdoba. 